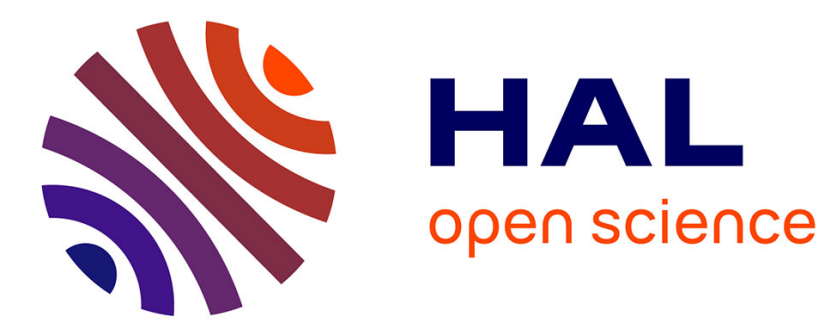

\title{
Staggered vertical self-organization of stacked InAs/InAlAs quantum wires on InP 001
}

J. Brault, Michel Gendry, O. Marty, M. Pitaval, J. Olivares, G. Grenet, G. Hollinger

\section{- To cite this version:}

J. Brault, Michel Gendry, O. Marty, M. Pitaval, J. Olivares, et al.. Staggered vertical self-organization of stacked InAs/InAlAs quantum wires on InP 001. Applied Surface Science, 2000, 162, pp.584-589. hal-02190195

\section{HAL Id: hal-02190195 \\ https://hal.science/hal-02190195}

Submitted on 22 Jul 2019

HAL is a multi-disciplinary open access archive for the deposit and dissemination of scientific research documents, whether they are published or not. The documents may come from teaching and research institutions in France or abroad, or from public or private research centers.
L'archive ouverte pluridisciplinaire HAL, est destinée au dépôt et à la diffusion de documents scientifiques de niveau recherche, publiés ou non, émanant des établissements d'enseignement et de recherche français ou étrangers, des laboratoires publics ou privés. 


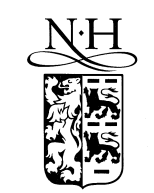

ELSEVIER
Applied Surface Science 162-163 (2000) 584-589 applied

surface science

www.elsevier.nl/locate/apsusc

\title{
Staggered vertical self-organization of stacked InAs /InAlAs quantum wires on $\operatorname{InP}(001)$
}

\author{
J. Brault ${ }^{\mathrm{a}, *}$, M. Gendry ${ }^{\mathrm{a}}$, O. Marty ${ }^{\mathrm{b}}$, M. Pitaval ${ }^{\mathrm{b}}, \mathrm{J}_{\text {. Olivares }}{ }^{\mathrm{c}}$, G. Grenet $^{\mathrm{a}}$, \\ G. Hollinger ${ }^{\text {a }}$ \\ a Laboratoire d'Electronique-LEOM, Ecole Centrale de Lyon, CNRS-UMR 5512, 36 Av G. de Collongue, F-69131 Ecully Cedex, France \\ b Laboratoire d'Electronique-LENAC, Université Claude Bernard-Lyon 1, F-69622 Villeurbanne Cedex, France \\ ${ }^{\mathrm{c}}$ Laboratoire de Physique de la Matière-LPM, UMR CNRS 5511, INSA de Lyon, Bât. 502, 69621 Villeurbanne Cedex, France
}

\begin{abstract}
Using atomic force microscopy (AFM) imaging, transmission electron microscopy (TEM) and photoluminescence (PL), we have studied InAs stacked islands on InP(001) versus the InAlAs spacer layer thickness (SLT). We have found that first wire-like island shape is strongly favored by such a stacking process and second in the 10-25 nm SLT range, the wire size and height are dependent on the SLT. TEM images show off a new surprising staggered vertical island organization that can be explained by the phase separation appearing in the InAlAs spacer layers. C) 2000 Elsevier Science B.V. All rights reserved.
\end{abstract}

Keywords: Quantum wires; Staggered vertical self-organization; InAs/InAlAs/InP; Solid-source MBE; AFM; TEM; Photoluminescence

\section{Introduction}

For the last few years, the self-formation of strained coherent islands using Stranski-Krastanov growth mode has drawn an increasing attention because it had emerged as a powerful way of fabricating nanoscale structures in which a lateral confinement of carriers can be obtained adding new functionality to devices [1]. In this context, InAs dots on InP [2-4] is a system of technological interest for the realization of low-threshold lasers operating in the $1.55-\mu \mathrm{m}$ range wavelength. Recently, this system

\footnotetext{
* Corresponding author. Tel.: +33-047-2186086; fax +33-0478433593.

E-mail address: julien.brault@ec-lyon.fr (J. Brault).
}

has been also revealed promising to fabricate infrared photodetectors because of a strong intraband absorption in normal incidence configuration [5]. However, an actual control during growth of the dot homogeneity (size, shape, spacing and even composition) still remains a technological challenge. In this respect, a widely used approach [6-8] to improve the nanostructure size shape and spacing uniformity is to achieve stacked structures having alternating layers of coherently strained nanostructure layers and spacer layers. In such a stacking process, each successive spacer layer acts as a filter leading to a more homogenous dot population in the newly growing dot layer than in the previously buried one. Furthermore, it is commonly agreed [6,7], that such a stacking process induces an on-top vertical dot arrangement because islands nucleate where the mismatch be- 
tween island and spacer layer surface is reduced by the strain due to a buried island.

In this paper, we will show that in IInAs/ InAlAs $\}_{n} / \mathrm{InP}(001)$ stacked structures, (i) the stacking process produces well-organized InAs wires and (ii) that the usual on-top vertical island organization does not exist any longer when the spacer layer is an immiscible alloy as InAlAs but is replaced by a new surprising staggered vertical island organization. As a matter of fact, in the case of InAlAs spacer layers, the InAlAs surface can undergo a composition modulation due to alloy demixing. Such a surface composition modulation can overpower the direct elastic effect due to the buried strained island. This effect is thought to be the reason for the remarkable staggered vertical arrangement of stacked wire-like islands we observed. Finally, note that this newly found spacer layer effect acts as a filter (as the classical one) for enhancing the wire self-organization as issued from atomic force microscopy (AFM) and photoluminescence (PL) measurements.

\section{Experimental}

Hence, we have studied stacked structures made of five strained InAs island layers separated by $\mathrm{In}_{0.52} \mathrm{Al}_{0.48} \mathrm{As}$ spacer layers lattice-matched (LM) on $\mathrm{InP}(001)$ substrate as a function of the spacer layer thickness (SLT): 2.1, 5, 10, 15, 25 and $40 \mathrm{~nm}$. The LM InAlAs is an alloy well known for undergoing alloy demixing at usual growth temperature because of its intrinsic surface roughness [9]. The InAs deposit was fixed at a 3-monolayer (ML) equivalent thickness because it is just above the critical threshold for the $2 \mathrm{D} / 3 \mathrm{D}$ growth mode transition (2.5 ML) as measured by reflection high-energy electron diffraction (RHEED). The non-capped fifth InAs layer in-plane morphology has been imaged using ex-situ AFM, while transmission electron microscopy (TEM) images were performed to characterize island vertical arrangement. AFM study was carried out using a Park Scientific Instruments microscope, model $\mathrm{CP}$, operating in the contact mode. The TEM images were recorded on cross-section (X-TEM) preparations along [1-10] zone axis using a TOPCON EM-002B analytical and ultrahigh resolution transmission electron microscope working at
$200 \mathrm{kV}$ with $0.18 \mathrm{~nm}$ point-to-point resolution. Conventional dark-field images using planes (002) were obtained. Considering the sample thickness in the 100 -nm range, the wave intensity is weak due to a low structure factor (extinction distance exceeds 1 $\mu \mathrm{m})$ corresponding to kinematic conditions. This implies that intensity is quite constant around Bragg orientation and that plane distortions have low influences. On the contrary, since the structure factor is very sensitive to a composition change, the wave intensity and the image contrast are highly depending on aluminium and indium composition. In summary, these conditions allow a good composition contrast with low plane distortion influences. Finally, PL measurements have been performed to fully quantify the vertical and lateral homogeneity of the grown self-organized island stacked layers. They were performed using an $\mathrm{AlGaAs}$ laser diode emitter $(\lambda=800 \mathrm{~nm})$ and liquid nitrogen-cooled $\mathrm{InGaAs}$ detector.

The samples were grown by solid-source molecular beam epitaxy (MBE) on semi-insulating $\operatorname{InP}(001)$ in a Riber 2300 reactor. The substrate temperature was measured using an infrared pyrometer previously calibrated from the melting point $\left(525^{\circ} \mathrm{C}\right)$ of InSb. After thermal desorption of the native oxide on the InP(001) substrates, a 400-nm LM InAlAs buffer layer was grown. The growth temperature was fixed at $525^{\circ} \mathrm{C}$ and the LM InAlAs buffer and spacer layers were grown at a $1 \mu \mathrm{m} / \mathrm{h}$ growth rate and a $\mathrm{V} / \mathrm{III}$ beam equivalent pressure (BEP) ratio of 20 . The InAs growth conditions were chosen to be as close as possible to thermodynamic equilibrium. The $3 \mathrm{ML}$ of InAs were grown at a reduced growth rate of $0.25 \mu \mathrm{m} / \mathrm{h}\left(0.22 \mathrm{ML} \mathrm{s}^{-1}\right)$ and the arsenic pressure was fixed at $\sim 1-3 \times 10^{-6}$ Torr. After each InAs deposit, the samples were held for $2 \mathrm{~min}$ at growth temperature and then covered with a LM InAlAs spacer. However, the last InAs layer of some samples has been left uncapped to perform AFM measurements. Finally, two samples have been grown as reference: both of them contain a single island layer (i.e. $3 \mathrm{ML}$ InAs on InAlAs/InP(001)) grown within the same conditions as those used in the stacked structures but the first one (A1) had been kept uncapped and the second (A2) had been capped with $300 \mathrm{~nm}$ LM InAlAs layer, for AFM and PL measurement requirements, respectively. 


\section{AFM results}

Typical AFM images are shown in Fig. 1 while sample labels and corresponding main results are collected in Table 1. Firstly, note that the tendency for wire-like shape (elongated along the [1ํㅣㅇㅣ direction) observed for all samples (even for the A1 case) is a typical feature of InAs dots grown on InA$1 \mathrm{As} / \mathrm{InP}(001)$ [10]. It is attributed to intrinsic InAlAs alloy surface roughness inducing anisotropy in surface diffusion and/or reactivity at step edges. In Fig. 1 , one can clearly observe that this wire-like shape is strongly improved by stacking dot layers and that the shape, size and lateral distribution of the wires are strongly dependent on the InAlAs SLT. The best wire homogeneity (from shape, size as well as spac-
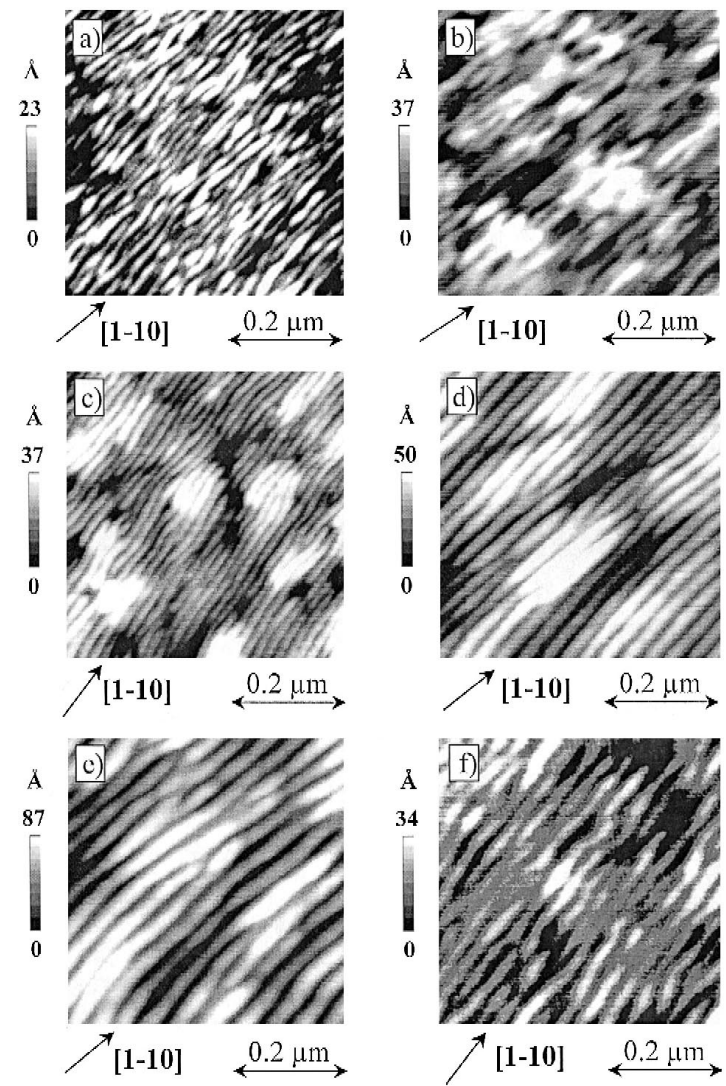

Fig. 1. Typical AFM images of the InAs island morphology of (a) a reference single-layer sample of $3 \mathrm{ML}$ InAs/InAlAs/InP(001), and of five stacked InAs layer samples with spacer layer thickness equal to (b) 5 , (c) 10 , (d) 15 , (e) 25 and (f) $40 \mathrm{~nm}$. ing point of view) is found for samples D-F, viz., for the [10-25 nm] SLT range. For smaller SLT (samples B and C), the spacer layer filtering effect does not act so efficiently: the islands' shape and width remain almost similar in size and dispersion than those observed for non-stacked islands (sample A1). We just note an increase in height $(1.6 \mathrm{~nm}$ instead of $0.8 \mathrm{~nm}$ ). For samples D-F, almost perfect wires with micrometric length are obtained. Moreover, for SLT equal to $10 \mathrm{~nm}$ (sample D), wire height and width are comparable to those of the non-stacked case (sample A1), while from 10 to 25 $\mathrm{nm}$ SLT (samples D-F), the wire size increases continuously in height and width. Finally, for $40 \mathrm{~nm}$ SLT (sample G), the stacking effect does not hold any more and the island shape looks like that obtained on sample A1 but with a slightly more wirelike shape. At this latter stage, there is no more interaction between growing and buried islands. To summarize, from an AFM imaging point of view, InAs wire stacking process with InAlAs spacer layers is in good agreement with the theoretical predictions by Tersoff et al. [7] about wire lateral spacing versus SLT. However, we will see in the next item, viz. from a TEM point of view, that the process leading to this well organization cannot originate from the same physical process, i.e. the strain effect associated to previously buried island.

\section{TEM results}

Typical cross-section TEM images are shown in Fig. 2a-c. Fig. $2 a$ and $b$ has been performed on the just-described samples C and D (5 and $10 \mathrm{~nm} \mathrm{SLT),}$ while Fig. 2c corresponds to an extra sample grown with a very broad SLT of $50 \mathrm{~nm}$. No sharp contrast revealing dislocations is observed on all the samples: they are dislocation-free at the field of view scale. In Fig. 2a and b, one can clearly see a remarkable staggered wire vertical arrangement, found to be optimum when the self-organization described in the previous paragraph is maximum (sample D). In addition, the InAlAs spacer layer seems to wave to mimic the island shape. Finally, when comparing total dot volume (per unit length), either from AFM or from TEM measurements, with the actual InAs 
Table 1

AFM and PL results versus SLT for stacked structures made of five strained InAs layers separated by $\operatorname{In}_{0.52} \mathrm{Al}_{0.48} \mathrm{As}$ spacer layers on InP(001) substrate

\begin{tabular}{|c|c|c|c|c|c|c|c|}
\hline \multicolumn{2}{|l|}{ Sample } & \multicolumn{4}{|l|}{ AFM } & \multicolumn{2}{|l|}{ PL } \\
\hline Sample name & SLT (nm) & Height (nm) & Width (nm) & $\begin{array}{l}\text { Density } / \\
(\mu \mathrm{m} \times \text { length })\end{array}$ & Shape and organization & $\begin{array}{l}E_{\mathrm{g}} \\
(300 \mathrm{~K}) \\
(\mathrm{meV})\end{array}$ & $\begin{array}{l}\text { Full } \\
\text { width at half } \\
\text { maximum } \\
\text { (FWHM) (meV) }\end{array}$ \\
\hline $\mathrm{A} 1$ or $\mathrm{A} 2$ & no & $0.8 \pm 0.4$ & $18 \pm 5$ & $\sim 60$ & Uncertain & 870 & 156 \\
\hline $\mathrm{B}$ & 2.1 & $1.6 \pm 0.8$ & $18 \pm 5$ & $\sim 60$ & Uncertain & 729 & 286 \\
\hline $\mathrm{C}$ & 5 & $1.6 \pm 1$ & $18 \pm 6$ & $\sim 60$ & Uncertain & 701 & 333 \\
\hline $\mathrm{D}$ & 10 & $0.8 \pm 0.3$ & $18 \pm 3$ & $\sim 60$ & Well-organized wire & 848 & 131 \\
\hline $\mathrm{E}$ & 15 & $1.6 \pm 0.5$ & $27 \pm 3$ & $\sim 40$ & Well-organized wire & 813 & 139 \\
\hline $\mathrm{F}$ & 25 & $3 \pm 1$ & $35 \pm 5$ & $\sim 30$ & Well-organized wire & 799 & 150 \\
\hline G & 40 & $1.3 \pm 0.6$ & $30 \pm 6$ & $\sim 35$ & Uncertain & 863 & 133 \\
\hline
\end{tabular}

deposited amount, a ratio lesser than 1 is found. One can, however, easily see that such a lacking InAs amount can be completely retrieved as a thin InAs wetting layer (light color) laying below the wires.

Looking now at Fig. 2c, one can observe within the spacer layer light- or dark-colored features corresponding to indium-rich or aluminum-rich regions, respectively, and outcoming from InAlAs phase separation. Indium-rich V-like features (light color) originate from each wire and propagate evanescently through the InAlAs spacer layer. In other words, each InAs wire facets are extended within the InAlAs spacer layer by an indium-rich region, while InAs wire-top and in-between wires spacing by an
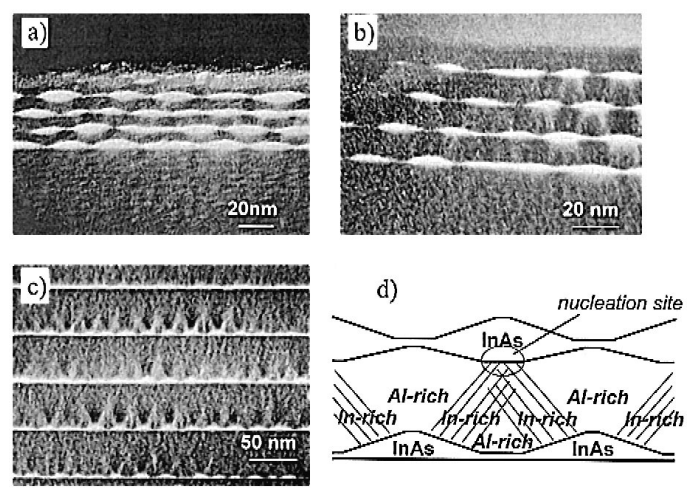

Fig. 2. Cross-sectional ( $g=(200)$ dark-field) TEM images showing the vertical arrangement of five stacked InAs layer samples with spacer layer thickness equal to (a) 5, (b) 10 and (c) $50 \mathrm{~nm}$. (d) A schema showing the best nucleation sites when alloy demixing is taken into account. aluminum-rich region. When two V-like arms (indium-rich region) meet, they generate an adequate site for the nucleation of a new InAs wire in the next plane of a stacked structure. This meeting point may be estimated to be at a thickness between 10 and 30 $\mathrm{nm}$ from the wire plane corresponding to approximately the wire width. This idea has been schematized in Fig. 2d.

Let us make some comments about InAlAs alloy phase separation. Firstly, one has to bear in mind that LM InAlAs alloy can undergo phase separation only if surface presents some roughness [9]. Indeed, this condition is fulfilled in our case because of the previous InAs island growth, and the indium-rich regions disappear when the SLT allows a surface smoothening. Second, this phase separation has been shown to be strongly dependent on growth temperature and to reach a maximum for the range 400 $450^{\circ} \mathrm{C}$. At these low-growth temperatures, the clusters are typically $2 \mathrm{~nm}$ wide along the [110] direction. At the current growth temperature $525^{\circ} \mathrm{C}$, the phase separation is normally not detectable by TEM. However, it is now well established [11-15] that when a material is stressed because it is coherently deposited on a different material layer (the island InAs layer in our case), its total energy can be lowered by an adequate roughening of its surface (the spacer layer waving in our case) or, if this material is an immiscible alloy, by an adequate demixing. Thus, there exists a complex interdependence between phase separation, surface morphology and mismatch stresses whose combined action is 


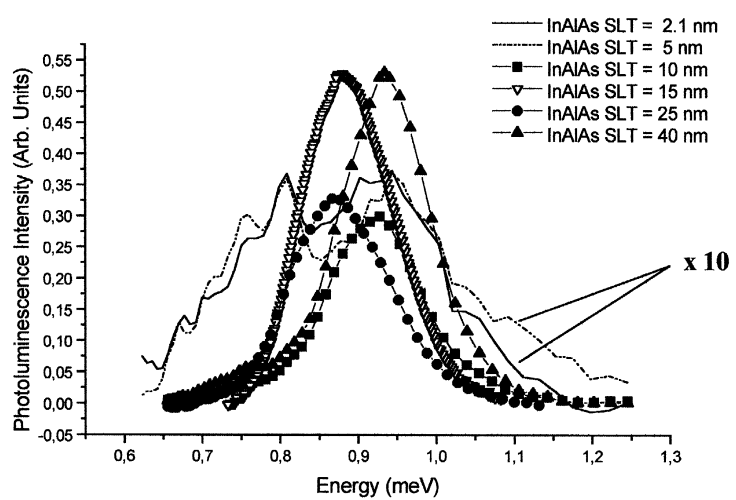

Fig. 3. Comparison of $300 \mathrm{~K}$ PL spectra of five stacked InAs layer samples with different spacer layer thickness.

thought to be at the root of the observed surprising island staggered vertical organization.

\section{PL results}

The room temperature PL spectra are reported in Fig. 3. The corresponding PL peak characteristics, i.e. the gap $\left(E_{\mathrm{g}}\right)$ at $300 \mathrm{~K}$ defined as the lower energy at half maximum and FWHM, are collected in Table 1. Sample A2 has been capped by LM InAlAs up to $300 \mathrm{~nm}$ to compare with the other five InAs layer samples. $E_{\mathrm{g}}$ at $300 \mathrm{~K}$ is found at 870 $\mathrm{meV}$ with a FWHM equal to $156 \mathrm{meV}$. For samples $\mathrm{B}$ and $\mathrm{C}$, their very broad PL peaks (FWHM equal to $286 \mathrm{meV}$ and $333 \mathrm{meV}$, respectively) can be thought as resulting from the large size distribution and lack of vertical self-organization of their involved InAs wire layers. For samples D, E and F, $E_{\mathrm{g}}$ at $300 \mathrm{~K}$ are at $848 \mathrm{meV}, 813 \mathrm{meV}$ and $799 \mathrm{meV}$, respectively, and thus shifting toward lower energies in perfect accordance with the widening of the wire size and thus lowering of the quantum confinement. Their smaller FWHMs (around 130-150 meV) clearly take its origin in the good wire self-organization as found from AFM for these samples. The smaller FWHM measured for the sample D (131 meV) seems to indicate that the best wire self-organization is obtained for the 10-nm SLT as given by forewarning TEM observations. Finally, for sample G, where the influence of the wire size is no more correlated with the SLT, the $E_{\mathrm{g}}$ at $300 \mathrm{~K}$ is found at $863 \mathrm{meV}$ confirming that the wire size is reduced compared to sample F. In summary, the PL measurements well confirm the self-organization improvement supplied by a stacking process observed by AFM and TEM imaging.

\section{Concluding remarks}

From this study, we have found that a stacking process enhances the wire-like shape of the nanostructures obtained in $\{\mathrm{InAs} / \mathrm{InAlAs}\}_{n} / \mathrm{InP}(001)$. Moreover, such a stacking process leads in our case to a remarkable staggered vertical alignment of the wires instead of the usual on-top vertical alignment. This arrangement is thought to be the result of complex interaction in between (i) the stress field from each InAs island layer within each InAlAs spacer layer, (ii) the phase separation appearing in the InAlAs spacer layer and (iii) the surface morphology. In addition, such a wire vertical and lateral organizations depend on many parameters that are not independent and that must be judiciously chosen to improve the size homogeneity and the staggered vertical alignment of the wires essential for actual applications.

\section{Acknowledgements}

This work has been supported by the "Région Rhône-Alpes" under the contracts no. 97021186 and no. 97021195.

\section{References}

[1] D. Bimberg, M. Grundmann, N.N. Ledentsov, MRS Bull. (1998) 31, February.

[2] S. Fafard, Z. Wasilewski, J. McCaffrey, S. Raymond, S. Charbonneau, Appl. Phys. Lett 68 (1996) 991.

[3] V.M. Ustinov, E.R. Weber, S. Ruminov, Z. Liliental-Weber, A.E. Zhukov, A.Yu. Egorov, A.R. Kovsh, A.F. Tsatsul'nikov, P.S. Kop'ev, Appl. Phys. Lett. 72 (1998) 362.

[4] B. Lambert, A. Le Corre, V. Drouot, H. L'Haridon, S. Loualiche, Semicond. Sci. Technol. 13 (1998) 143.

[5] A. Weber, O. Gauthier-Lafaye, F.H. Julien, J. Brault, M. Gendry, Y. Desières, T. Benyattou, Appl. Phys. Lett. 74 (1999) 413. 
[6] Q. Xie, A. Madhukar, P. Chen, N.P. Kobayashi, Phys. Rev. Lett. 75 (1995) 2542.

[7] J. Tersoff, C. Teichert, M.G. Lagally, Phys. Rev. Lett. 76 (1996) 1675.

[8] I. Mukhametzhanov, R. Heitz, J. Zeng, P. Chen, A. Madhukar, Appl. Phys. Lett. 73 (1998) 1841.

[9] C. Priester, G. Grenet, JVSTB 16 (1998) 2421.
[10] J. Brault, M. Gendry, G. Grenet, G. Hollinger, Y. Desières, T. Benyattou, Appl. Phys. Lett. 73 (1998) 2932.

[11] J.E. Guyer, P.W. Voorhees, Phys. Rev. Lett. 74 (1995) 4031.

[12] J. Tersoff, Phys. Rev. Lett. 77 (1996) 2017.

[13] F. Glas, Phys. Rev. B 55 (1997) 11277.

[14] F. Leonard, R.C. Desai, Phys. Rev. B 56 (1997) 4955.

[15] F. Leonard, R.C. Desai, Phys. Rev. B 57 (1998) 4805. 